\title{
Synthesis and photocatalytic activity of hierarchical flower-like $\mathrm{SrTiO}_{3}$ nanostructure
}

\author{
Xiao-Yong Lai ${ }^{1 \dagger}$, Cheng-Rui Wang ${ }^{2 \dagger}$, Quan Jin ${ }^{1}$, Ran-Bo Yu ${ }^{2}$ and Dan Wang ${ }^{1 *}$
}

\begin{abstract}
A hierarchical flower-like $\mathrm{SrTiO}_{3}$ nanostructure was synthesized by a simple and direct hydrothermal method. The products were characterized by $\mathrm{X}$-ray diffraction, scanning electron microscopy, and transmission electron microscopy. Structure characterizations suggest that the as-synthesized $\mathrm{SrTiO}_{3}$ crystal has eight symmetric branches growing along the $\langle 111\rangle$ direction and each branch has many ordered small laminae, forming a well-defined flower-like structure. By adjusting the $\mathrm{pH}$ value of the reaction system, the morphology could be changed continuously from flower-like structure to cube, then to sphere. The hierarchical flower-like $\mathrm{SrTiO}_{3}$ nanostructure exhibits a higher photocatalytic activity for degrading Rhodamine B than its cubic and spherical counterparts.
\end{abstract}

In recent years, nanostructured materials have attracted considerable interest because of their unique physical and chemical properties as well as potential applications in various electronic devices [1-17]. It is well-known that the morphology and microstructure of the inorganic materials strongly influence their properties, such as isotropic or anisotropic behavior and region-dependent surface reactivity $[4,18]$. Therefore, great effort has been devoted to synthesizing various inorganic materials with controlled shapes and complex assembled architectures, which are expected to offer superior properties for many applications in optics [15,19], electronics [20], magnetics [21,22], medicines [23], biology [24] and so on [25-29]. During the past decades, a number of novel nanostructured materials with various shapes, such as nanorods, nanocubes, nanobelts, nanowires, nanofibers, nanotubes, and dendrites, have been reported. However, it is still a challenge to understand and predict the final architecture of the nanoscale building blocks and construct more complex hierarchical threedimentional (3D) architectures.

Strontium titanate $\left(\mathrm{SrTiO}_{3}\right)$, as an important perovskite conductor, has many potential applications in various microelectronic heterostructures [30], oxygen sensors [31], solar cells [32], and thermoelectric devices [33], due to its excellent dielectric, photoelectric, optical and catalytic properties. Moreover, $\mathrm{SrTiO}_{3}$ with a band gap similar to that of titania, is also a very promising photocatalyst because of its superior photocorrosion resistibility, thermal stability, and easily doping to control the electrical properties [34-37]. Conventional solid-state reaction method for $\mathrm{SrTiO}_{3}$ typically requires high reaction temperature above $900^{\circ} \mathrm{C}$ and usually results in the agglomeration of the particles with different sizes and impurity derived from incomplete reaction [38]. Recently, hydrothermal methods have attracted much attention due to high purity of their products and various $\mathrm{SrTiO}_{3}$ nanostructured materials have been synthesized, such as nanocubes [38], nanotubes [33], nanotube-arrays [21], nanofibers [20], dendrites [15,39] and multipod crystal [40].

Herein, we report a flower-like $\mathrm{SrTiO}_{3}$ nanostructure with eight symmetric branches growing along the $\langle 111\rangle$ direction. The shapes of $\mathrm{SrTiO}_{3}$ crystals evolved from flower-like structure to cube, then to sphere by adjusting the $\mathrm{pH}$ value of the reaction system. Photocatalytic measurement for degrading Rhodamine B showed the high activity of the flower-like $\mathrm{SrTiO}_{3}$ nanostructure, superior over its cubic and spherical counterparts.

Fig. 1 shows the X-ray diffraction (XRD) pattern of the

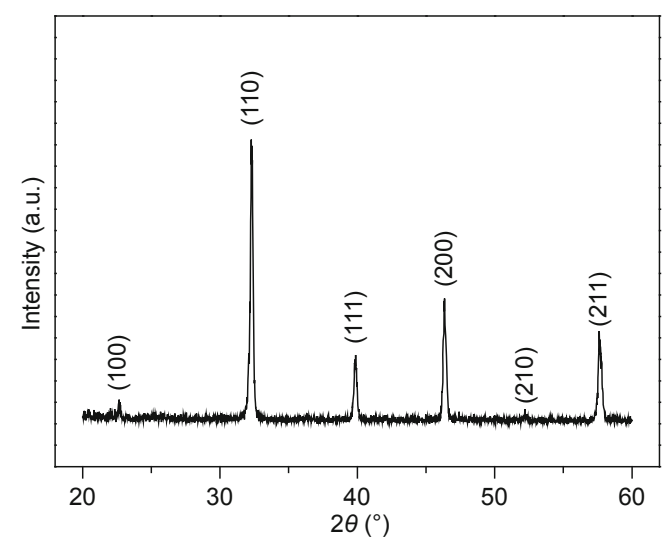

Figure 1 XRD pattern of the flower-like $\mathrm{SrTiO}_{3}$ nanostructure.

\footnotetext{
${ }^{1}$ State Key Laboratory of Multiphase Complex Systems, Institute of Process Engineering, Chinese Academy of Sciences, Beijing 100190, China

${ }^{2}$ Department of Physical Chemistry, University of Science and Technology Beijing, Beijing 100083, China

${ }^{\dagger}$ These authors contributed equally to this work.

*Corresponding author (email: danwang@ipe.ac.cn)
} 
as-prepared flower-like $\mathrm{SrTiO}_{3}$ nanostructure. The diffraction pattern distinctly indicates the high crystallinity of the products. The diffraction peaks of the products can be indexed as cubic $\mathrm{SrTiO}_{3}$ with a lattice constant of $a=$ $3.920 \AA$, which is in good agreement with the literature value (JCPDS Card No. 35-0734, $a=3.905 \AA$ ). No peaks of impurities are detected, revealing the high purity of the as-synthesized products.

Scanning electron microscopy (SEM) and transmission electron microscopy (TEM) were used to investigate the morphological and microstructural features of the as-synthesized $\mathrm{SrTiO}_{3}$ (Fig. 2). Fig. 2a shows that the product consists almost of such flower-like structures with an average size of $0.8 \mu \mathrm{m}$, indicating that crystals of high yield and good uniformity can be achieved with this simple and easily controlled approach. The high magnification images (Figs $2 \mathrm{~b}-\mathrm{d}$ ) show the morphology of an individual flower-like crystal viewed from frontal, flank, and tilted frontal perspectives, respectively. All the SEM images show its 3D structure, consisting of a central cube with eight symmetric branches emerging away from the apexes. Each branch is composed of a stack of small laminas assembled along the branch axis and slightly slid with respect to one another, forming a well-defined flower-like $\mathrm{SrTiO}_{3}$ nanostructure. The average length of the branches is $\sim 0.5 \mu \mathrm{m}$ and every four adjacent branches form a flower-like structure. The
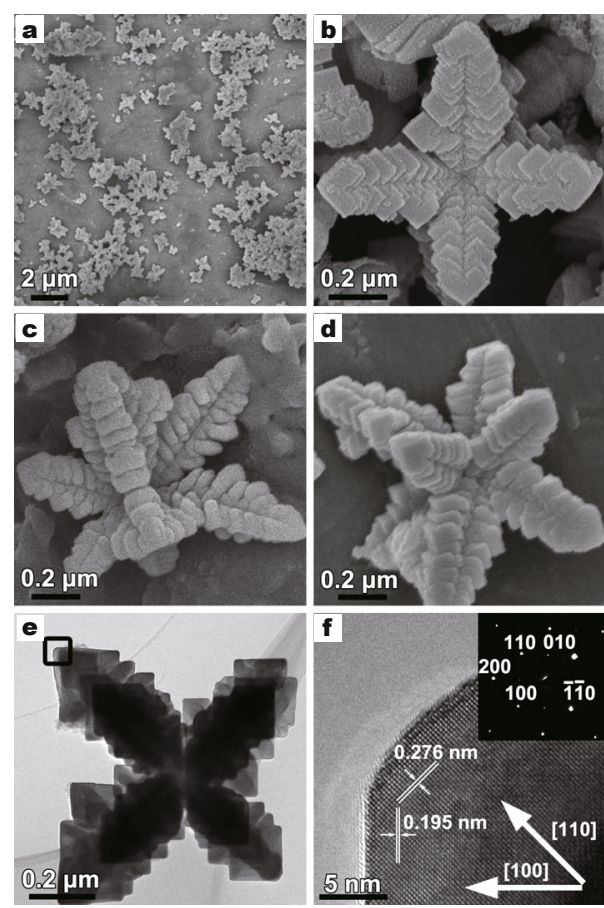

Figure 2 SEM (a-d), TEM (e) and HRTEM (f) images of $\mathrm{SrTiO}_{3}$ products. The morphology of an individual flower-like crystal was observed from (a) frontal, (b) flank and (c) tilted frontal perspectives, respectively. The inset in (f) is the corresponding SAED pattern. high symmetry and single crystallinity is revealed by TEM results. The TEM images of an individual flower-like crystal is shown in Fig. 2e, demonstrating a 4-fold symmetric structure like a flower. In fact, it has eight branches with four pointing above and four standing on the $\mathrm{Cu}$ grid but hidden below. Further structural characterization of the crystal was carried out by high resolution TEM (HRTEM). Fig. $2 \mathrm{f}$ is an HRTEM image taken from the crystal in Fig. $2 \mathrm{e}$, exhibiting the lattice structure at the tip of a branch, which has clear lattice fringes indicating its single crystallinity nature. The regular spacing of the observed lattice planes is about 0.276 and $0.195 \mathrm{~nm}$, which are, respectively, consistent with the [110] and [200] lattice spacing of the cubic $\mathrm{SrTiO}_{3}$. The two-dimensional (2D) exposed surface and four edges of $\mathrm{SrTiO}_{3}$ laminae can also be determined to be $(001),(200),(0 \overline{2} 0),(\overline{2} 00)$ and $(020)$ planes, respectively. The corresponding selected-area electron diffraction (SAED) pattern (inset of Fig. 2f), recorded along the [001] zone axis, further confirms its single-crystalline structure and also suggests that the preferentially oriented crystal at the interfaces with [001] zone axis nearly parallel to the incident electron beam. Moreover, the SAED patterns corresponding to the other trunk tips are observed to be exactly same, which suggests that the whole crystal is a singlecrystal. Considering the symmetry of the eight branches, it can be concluded that each branch grows along the $\langle 111\rangle$ direction.

The time-dependent shape evolution provides the experimental evidence on how the flower-like structure formed (Fig. 3). At the early stage of hydrothermal reaction (60 min), only aggregated irregular nanoparticles are observed (Fig. 3a), and XRD pattern (Fig. 3d) confirms the amorphous nature of those irregular nanoparticles, which suggests that the crystallization does not happen. Prolong-
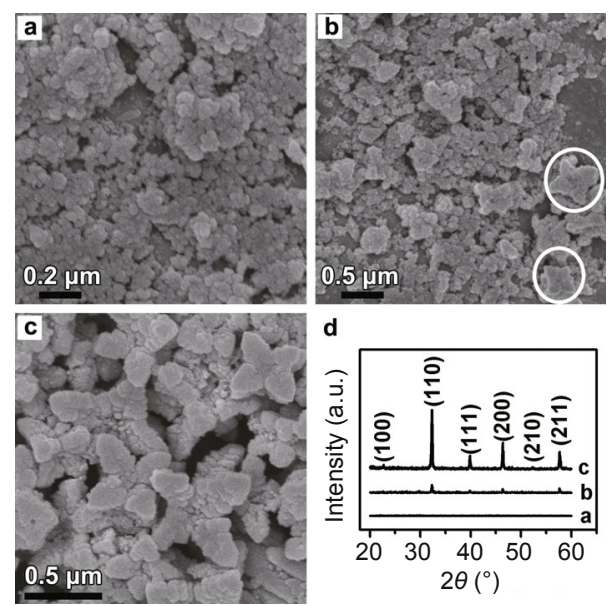

Figure 3 SEM images of $\mathrm{SrTiO}_{3}$ products obtained after different reaction times: (a) $60 \mathrm{~min}$, (b) $65 \mathrm{~min}$, (c) $90 \mathrm{~min}$ and (d) their corresponding XRD patterns. 
ing the reaction time to 65 min gives a few flower-like particles with short branches of $\sim 0.2 \mu \mathrm{m}$ (indicated by white open circles, Fig. 3b), besides those irregular nanoparticles. The corresponding XRD pattern exhibits the appearance of cubic $\mathrm{SrTiO}_{3}$ phase in the product, but the intensity of their diffraction peaks is comparably weak due to the lower proportion of the crystalline $\mathrm{SrTiO}_{3}$ in the product. As the reaction time was prolonged to $90 \mathrm{~min}$, XRD pattern (Fig. 3d) exhibits that the intensity of the $\mathrm{SrTiO}_{3}$ phases peaks significantly increases compared with those in the early stages. As shown in the SEM image (Fig. 3c), the proportion of flower-like $\mathrm{SrTiO}_{3}$ particles with branches increases as well as the length of the branches (from 0.2 to $0.3 \mu \mathrm{m}$ ). Further prolonging the reaction time to $24 \mathrm{~h}$, well-defined flower-like nanostructures could be obtained (Fig. 2).

Generally, the symmetry and shape of the crystals in the formation process is determined by their nucleation and subsequent growth. In the nucleation process, the crystalline phase of the crystal seeds is the most critical factor for forming special crystals. It is well known that $\mathrm{SrTiO}_{3}$ as a perovskite oxide crystallizes with a face-center-cubic (fcc) structure and the shape of fcc nanocrystals is mainly determined by the ratio of the growth rate in the $\langle 100\rangle$ to that in the $\langle 111\rangle$ directions [41]. In fcc structures, the $\{111\}$ facets typically has a higher energy than that of the $\{100\}$ facets due to the higher packing density and more under-coordinated atoms, which leads to a faster growth along the $\langle 111\rangle$ direction and a faster consumption of reaction agent, favoring the formation of cube-shaped crystal [42-43]. However, as the concentration of the reactant decreases due to the reaction consumption and the increasing size of crystal, the growth rate decreases and the mass transport of the reactant plays a critical role in the growth. Eight apexes of the cube have a significant advantage in receiving reactants over the $\{100\}$ facets, which further enhances the preferential kinetic growth along $\langle 111\rangle$ direction and leads to the formation of eight branches. The branching growth also generates a large surface area with many dangling bonds which allows the growth along $\langle 100\rangle$ direction on each branch and results in the formation of a stacking structure composed of small laminas. The formation of $\mathrm{SrTiO}_{3}$ under hydrothermal condition is thought to dehydrate from hydroxide [39]:

$$
\mathrm{Si}(\mathrm{OH})_{2}+\mathrm{Ti}(\mathrm{OH})_{4} \rightarrow \mathrm{SiTiO}_{3}+3 \mathrm{H}_{2} \mathrm{O} .
$$

For comparison, we increased the concentration of the reactant and enhanced the mass transport by adjusting the $\mathrm{pH}$ value of the original systems from 12.6 to 13.0, which facilitates the growth on a variety of crystal surfaces due to the larger mass transport rate and weakens the preference of the growth along $\langle 111\rangle$ direction, resulting in the formation of the cube-shaped $\mathrm{SrTiO}_{3}$ crystals (Fig. 4a).
Further increasing the $\mathrm{pH}$ value of the original systems to 13.4 , the high-energy faceted edges of the cube-shaped crystals encounter preferential dissolution, resulting in the formation of the spherical particles (Fig. 4b). Moreover, we also investigated the effect of reaction temperature on the growth (Fig. 5). The branches of the crystals obtained at higher reaction temperatures $\left(180\right.$ and $\left.200^{\circ} \mathrm{C}\right)$ significantly are short from 0.5 to $0.2 \mu \mathrm{m}$ (Figs $5 \mathrm{c}$ and $\mathrm{d}$ ) and their branch surfaces are smooth but lack of the clear edges compared with the crystal obtained at $160^{\circ} \mathrm{C}$, since the increase of the reaction temperature enhances the mass transport of the reactants and weakens the preference of the branch growth along $\langle 111\rangle$ direction. In contrast, $\mathrm{SrTiO}_{3}$ products obtained at $120^{\circ} \mathrm{C}$ show a rough branch surface and short branches (Fig. 5a), suggesting that the poor mass transport at low reaction temperature may restrain the crystal growth including along the $\langle 111\rangle$ direction. Based on these experimental evidences on the morphology evolution, the growth process of the flower-like nanostructure is simply described in Scheme 1.

The photocatalytic degradation of Rhodamine $\mathrm{B}$ was chosen as a model reaction to evaluate the photocatalytic activities of $\mathrm{SrTiO}_{3}$ products. Fig. 6 shows the degradation curves of Rhodamine $\mathrm{B}$ by pure $\mathrm{SrTiO}_{3}$ with different mor-
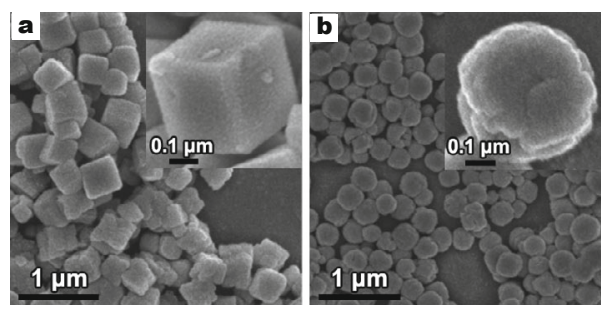

Figure 4 SEM images of $\mathrm{SrTiO}_{3}$ products obtained at different $\mathrm{pH}$ values of the original reaction solution: (a) 13.0 and (b) 13.4.

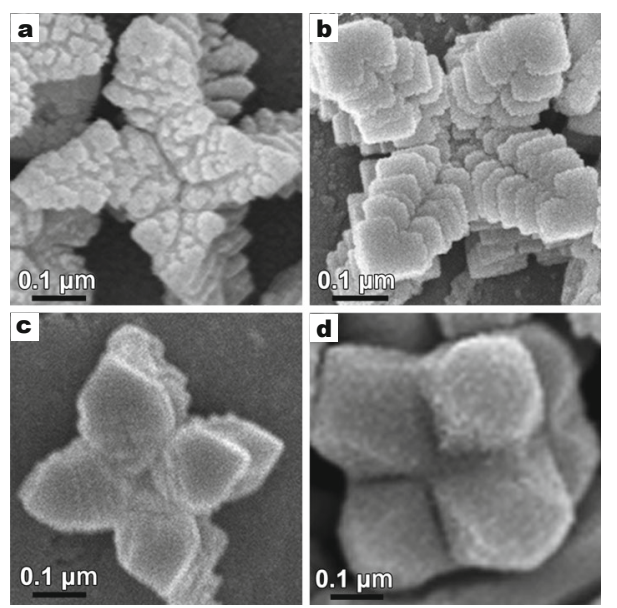

Figure 5 SEM images of $\mathrm{SrTiO}_{3}$ products obtained at different reaction temperatures: (a) $120^{\circ} \mathrm{C}$, (b) $160^{\circ} \mathrm{C}$, (c) $180^{\circ} \mathrm{C}$ and (d) $200^{\circ} \mathrm{C}$. 


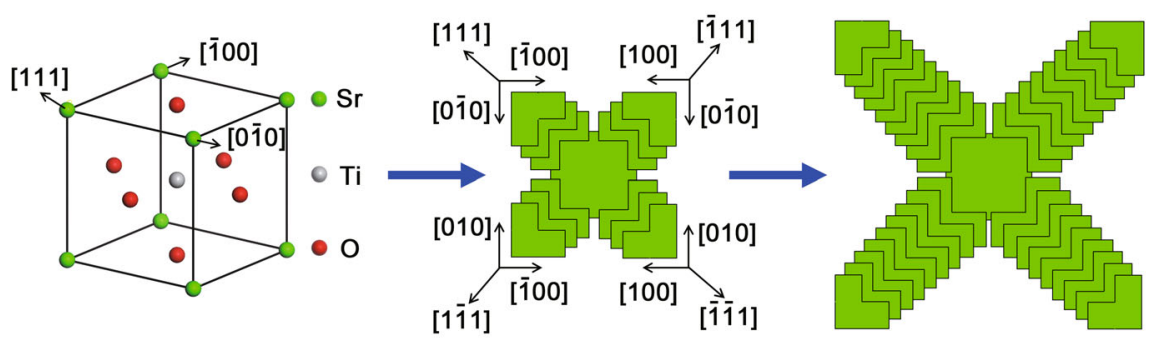

Scheme 1 Schematic illustration of the formation process of the hierarchical flower-like $\mathrm{SrTiO}_{3}$ nanostructure.

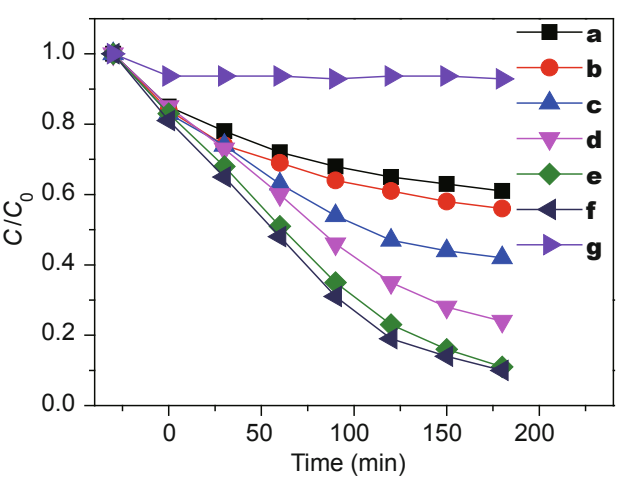

Figure 6 Photocatalytic degradation curves of Rhodamine B over different $\mathrm{SrTiO}_{3}$ products without and with $\mathrm{Ag}$ : (a, d) cubic $\mathrm{SrTiO}_{3}$, (b, e) spherical $\mathrm{SrTiO}_{3}$, (c, f) hierarchical flower-like $\mathrm{SrTiO}_{3}$, (g) without any $\mathrm{SrTiO}_{3}$ products.

phology. The hierarchical flower-like $\mathrm{SrTiO}_{3}$ nanostructure exhibits higher photocatalytic activities than other two $\mathrm{SrTiO}_{3}$ products (spherical and cubic). That may be because of the unique flower-like structure of the lattice, which makes them offer more surfaces for the photocatalytic reaction. In addition, the photocatalytic activities of $\mathrm{SrTiO}_{3}$ can be further enhanced by coupling with $\mathrm{Ag}$ and the resultant $\mathrm{Ag}-\mathrm{SrTiO}_{3}$ composite maintains their original morphologies. The enhancement of the photocatalytic activity is attributed to the formation of a Schottky barrier at the interface between $\mathrm{Ag}$ and the semiconductor $[35,44]$. Therefore, the photogenerated electrons in $\mathrm{SrTiO}_{3}$ would transfer to Ag, which acts as an electron trap promoting the separation of electrons and holes in $\mathrm{SrTiO}_{3}$. Thus, the photocatalytic activity of $\mathrm{SrTiO}_{3}$ can be efficiently improved by loading Ag nanoparticles. However, the photocatalytic activity of $\mathrm{Ag}-\mathrm{SrTiO}_{3}$ began to decrease with increasing $\mathrm{AgNO}_{3}$ concentration. The reason may be that large surface coverage of Ag particles leads to a decrease in the reaction probability of photon generated electrons and holes with adsorbed species on the surface, which is adverse to the photocatalytic activity of $\mathrm{SrTiO}_{3}$. More detailed investigation is still under way.
In summary, we successfully synthesized a flower-like $\mathrm{SrTiO}_{3}$ nanostructure. The unique stacking $\mathrm{SrTiO}_{3}$ crystals have eight symmetric branches along the $\langle 111\rangle$ directions, each of which shows a flower-like structure and consists of parallel $\mathrm{SrTiO}_{3}$ laminae. Appropriate $\mathrm{pH}$ value and reaction temperature are crucial for the formation of the unusual flower-like $\mathrm{SrTiO}_{3}$ crystals. This approach can be extended as an effective synthetic method for hierarchical, single-crystalline nanostructures of other inorganic semiconductors.

Received 3 February 2015; accepted 9 March 2015; published online 17 March 2015

1 Cui Y, Wei Q, Park H, Lieber CM. Nanowire nanosensors for highly sensitive and selective detection of biological and chemical species. Science, 2001, 293: 1289-1292

2 Huang $\mathrm{MH}$, Mao S, Feick $\mathrm{H}$, et al. Room-temperature ultraviolet nanowire nanolasers. Science, 2001, 292: 1897-1899

3 Xia YN, Gates B, Li ZY. Self-assembly approaches to three-dimensional photonic crystals. Adv Mater, 2001, 13: 409-413

4 Xia Y, Yang P, Sun Y, et al. One-dimensional nanostructures: synthesis, characterization, and applications. Adv Mater, 2003, 15: 353-389

5 Law M, Goldberger J, Yang P. Semiconductor nanowires and nanotubes. Annu Rev Mater Res, 2004, 34: 83-122

6 Lai X, Wang D, Han N, et al. Ordered arrays of bead-chain-like $\mathrm{In}_{2} \mathrm{O}_{3}$ nanorods and their enhanced sensing performance for formaldehyde. Chem Mater, 2010, 22: 3033-3042

7 Li Z, Lai X, Wang H, et al. Direct hydrothermal synthesis of singlecrystalline hematite nanorods assisted by 1,2-propanediamine. Nanotechnology, 2009, 20: 245603-245611

8 Mao D, Yao J, Lai X, et al. Hierarchically mesoporous hematite microspheres and their enhanced formaldehyde-sensing properties. Small, 2011, 7: 578-582

9 Huo ZY, Tsung CK, Huang WY, et al. Self-organized ultrathin oxide nanocrystals. Nano Lett, 2009, 9: 1260-1264

10 Bai F, Wang DS, Huo ZY, et al. A versatile bottom-up assembly approach to colloidal spheres from nanocrystals. Angew Chem Int Ed, 2007, 46: 6650-6653

11 Lai X, Li J, Korgel BA, et al. General synthesis and gas-sensing properties of multiple-shell metal oxide hollow microspheres. Angew Chem Int Ed, 2011, 50: 2738-2741

12 Wang DS, Xie T, Peng Q, Li YD. $\mathrm{Ag}, \mathrm{Ag}_{2} \mathrm{~S}$, and $\mathrm{Ag}_{2} \mathrm{Se}$ nanocrystals: synthesis, assembly, and construction of mesoporous structures. J Am Chem Soc, 2008, 130: 4016-4022 
13 Pan ZW, Dai ZR, Wang ZL. Nanobelts of semiconducting oxides. Science, 2001, 291: 1947-1949

14 Wang X, Zhuang J, Peng Q, Li Y. A general strategy for nanocrystal synthesis. Nature, 2005, 437: 121-124

15 Huang Y, Duan X, Lieber CM. Nanowires for integrated multicolor nanophotonics. Small, 2005, 1: 142-147

16 Mao Y, Banerjee S, Wong SS. Large-scale synthesis of single-crystalline perovskite nanostructures. J Am Chem Soc, 2003, 125 15718-15719

17 Zhu Z, Meng H, Liu W, et al. Superstructures and SERS properties of gold nanocrystals with different shapes. Angew Chem Int Ed, 2011, 50: 1593-11596

18 Li Y, Liu J, Huang X, Li G. Hydrothermal synthesis of $\mathrm{Bi}_{2} \mathrm{WO}_{6}$ uniform hierarchical microspheres. Cryst Growth Des, 2007, 7: 13501355

19 Gudiksen MS, Lauhon LJ, Wang J, Smith DC, Lieber CM. Growth of nanowire superlattice structures for nanoscale photonics and electronics. Nature, 2002, 415: 617-620

20 Duan X, Huang Y, Cui Y, Wang J, Lieber CM. Indium phosphide nanowires as building blocks for nanoscale electronic and optoelectronic devices. Nature, 2001, 409: 66-69

21 Chen H, Kolpak AM, Ismail-Beigi S. Electronic and magnetic properties of $\mathrm{SrTiO}_{3} / \mathrm{LaAlO}_{3}$ interfaces from first principles. Adv Mater, 2010, 22: 2881-2899

22 Jin J, Jiang L, Li TJ. Self-assembly of uniform spherical aggregates of magnetic nanoparticles through $\pi-\pi$ interactions. Angew Chem Int Ed, 2001, 40: 2135-2138

23 Pandey CM, Sumana G, Malhotra BD. Microstructured cystine dendrites-based impedimetric sensor for nucleic acid detection. Biomacromolecules, 2011, 12: 2925-2932

24 Xin Y, Jiang J, Huo K, Hu T, Chu PK. Bioactive $\mathrm{SrTiO}_{3}$ nanotube arrays: strontium delivery platform on Ti-based osteoporotic bone implants. ACS Nano, 2009, 3: 3228-3234

25 Dinh CT, Nguyen TD, Kleitz F, Do TO. Shape-controlled synthesis of highly crystalline titania nanocrystals. ACS Nano, 2009, 3 : 3737-3743

26 Greyson EC, Babayan Y, Odom TW. Directed growth of ordered arrays of small-diameter $\mathrm{ZnO}$ nanowires. Adv Mater, 2004, 16 : 1348-1352

27 Vayssieres L, Keis K, Hagfeldt A, Lindquist SE. Three-dimensional array of highly oriented crystalline $\mathrm{ZnO}$ microtubes. Chem Mater, 2001, 13: 4395-4398

28 Chu DW, Masuda Y, Ohji T, Kato K. Facile synthesis, characterization of $\mathrm{ZnO}$ nanotubes and nanoflowers in an aqueous solution. J Am Ceram Soc, 2010, 93: 887-893

29 Lu YF, Fan HY, Stump A, et al. Aerosol-assisted self-assembly of mesostructured spherical nanoparticles. Nature, 1999, 398: 223-226

30 Zhao K, Jin KJ, Huang Y, et al. Ultraviolet fast-response photoelectric effect in tilted orientation $\mathrm{SrTiO}_{3}$ single crystals. Appl Phys Lett, 2006, 89: 173507

$31 \mathrm{Hu}$ Y, Tan OK, Pan JS, Yao X. A new form of nanosized $\mathrm{SrTiO}_{3}$ material for near-human-body temperature oxygen sensing applicati- ons. J Phys Chem B, 2004, 108: 11214-11218

32 Yang S, Kou H, Wang J, Xue H, Han H. Tunability of the band energetics of nanostructured $\mathrm{SrTiO}_{3}$ electrodes for dye-sensitized solar cells. J Phys Chem C, 2010, 114: 4245-4249

33 Ohta H, Kim SW, Mune Y, et al. Giant thermoelectric Seebeck coefficient of a two-dimensional electron gas in $\mathrm{SrTiO}_{3}$. Nat Mater, 2007, 6: 129-134

34 Wang N, Kong D, He H. Solvothermal synthesis of strontium titanate nanocrystallines from metatitanic acid and photocatalytic activities. Powder Technol, 2011, 207: 470-473

35 Irie $\mathrm{H}$, Maruyama $\mathrm{Y}$, Hashimoto $\mathrm{K}$. $\mathrm{Ag}^{+}$- and $\mathrm{Pb}^{2+}$-doped $\mathrm{SrTiO}_{3}$ photocatalysts. A correlation between band structure and photocatalytic activity. J Phys Chem C, 2007, 111: 1847-1852

36 Konta R, Ishii T, Kato H, Kudo A. Photocatalytic activities of noble metal ion doped $\mathrm{SrTiO}_{3}$ under visible light irradiation. J Phys Chem B, 2004, 108: 8992-8995

37 Yin J, Ye JH, Zou ZG. Enhanced photoelectrolysis of water with photoanode $\mathrm{Nb}: \mathrm{SrTiO}_{3}$. Appl Phys Lett, 2004, 85: 689-691

$38 \mathrm{Xu} \mathrm{H}$, Wei S, Wang $\mathrm{H}$, et al. Preparation of shape controlled $\mathrm{SrTiO}_{3}$ crystallites by sol-gel-hydrothermal method. J Cryst Growth, 2006, 292: 159-164

39 Wang Y, Xu G, Yang L, et al. Formation of single-crystal $\mathrm{SrTiO}_{3}$ dendritic nanostructures via a simple hydrothermal method. J Cryst Growth, 2009, 311: 2519-2523

40 Toshima T, Ishikawa H, Tanda S, Akiyama T. Multipod crystals of perovskite $\mathrm{SrTiO}_{3}$. Cryst Growth Des, 2008, 8: 2066-2069

41 Wang ZL. Transmission electron microscopy of shape-controlled nanocrystals and their assemblies. J Phys Chem B, 2000, 104: 11531175

42 Lee SM, Jun YW, Cho SN, Cheon J. Single-crystalline star-shaped nanocrystals and their evolution: programming the geometry of nano-building blocks. J Am Chem Soc, 2002, 124: 11244-11245

43 Lee SM, Cho SN, Cheon J. Anisotropic shape control of colloidal inorganic nanocrystals. Adv Mater, 2003, 15: 441-444

44 Zhang Z, Feng J, Wang Z, et al. Guided growth of Ag nanoparticles on $\mathrm{SrTiO}_{3}$ (110) surface. J Chem Phys, 2011, 135: 144702-144706

Acknowledgements We thank Zhu ZY and Zhao HY's exploratory experiments. This research was supported by the National Natural Science Foundation of China (21031005, 20971125, 21006116, 20871015, 51072020 and 21050110428), and National Science Fund for Distinguished Young Scholars (21325105).

Author contributions Wang D designed the experiments; Lai XY and Wang CR performed the experiments and the data analysis with help from Yu RB and Jin Q, and Lai XY wrote the paper with support from Wang D. All authors contributed to the general discussion.

Conflict of interest The authors declare that they have no conflict of interest.

Supplementary information Experimental details are available in the online version of the paper. 


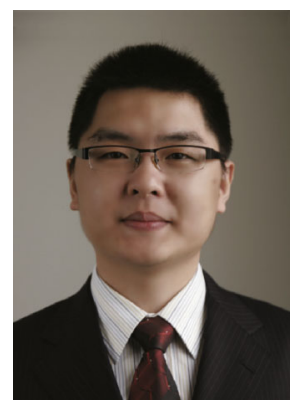

Xiao-Yong Lai received his BSc (2004) and PhD (2009) degrees from the Department of Materials and Science at Jilin University. Then, he moved to the Institute of Process Engineering, Chinese Academy of Sciences and joined Prof. Dan Wang's group. Now he is an associate researcher at the State Key Laboratory Cultivation Base of Natural Gas Conversion, Ningxia University. His research interests focus on the preparation of hollow and porous materials as well as their applications to photocatalysis, DSSCs and sensors.

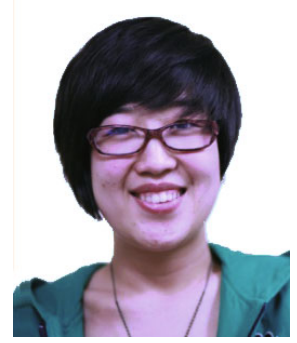

Quan Jin received her BSc degree (2005) at Jilin University, MSc degree (2008) at Beijing University of Chemical Technology and PhD degree (2012) at the University of Toyama. Then, she joined Prof. Dan Wang's group as an assistant professor at the State Key Laboratory of Multiphase Complex Systems, Institute of Process Engineering, Chinese Academy of Sciences. Her current research interests focus on the design and preparation of micro-/nanostructured functional materials as well as their application to photocatalysis, drug controlled release and biomedical materials.

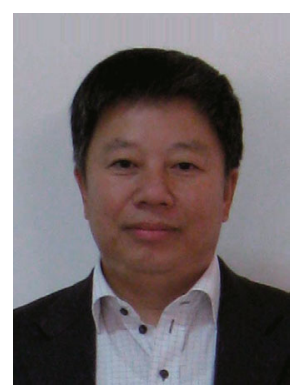

Dan Wang received his BSc and MSc degrees at Jilin University (1994 and 1997) and PhD degree at Yamanashi University (2001). In 2004, he was awarded the Hundred Talents Program of the Chinese Academy of Sciences and took his current position as a principal investigator at the State Key Laboratory of Multiphase Complex Systems, Institute of Process Engineering, Chinese Academy of Sciences. His research interests include inorganic material chemistry, surface and colloidal chemistry, hydrothermal chemistry, sol-gel chemistry and self-assembly processes, with focus on the design and synthesis of micro-/nanostructured functional inorganic materials and their applications in energy conversion and storage, photocatalysis and sensors.

中文摘要 本文报道了一种钛酸锶花状分级纳米结构. 实验结果表明该钛酸锶花状分级纳米结构沿钛酸锶晶体的〈111〉方向生长, 形成 八个分支结构, 每个分支结构由一系列纳米片堆叠而成, 整个粒子具有花状的形貌结构. 通过调节反应体系的 $\mathrm{pH}$, 可以得到纳米立方、 纳米球等其他形貌结构的钛酸锶晶体. 所合成钛酸锶对光催化降解罗丹明 B染料具有较高活性, 其中花状分级纳米结构钛酸锶的光催 化活性显著高于其他两种结构, 在光催化领域具有潜在应用前景. 